\title{
Photochemical Reaction of Nalidixic Acid in Methanol
}

\author{
Hyoung-Ryun Park, "Ok-Hyun Park, Hong-Yune Lee, Jung-Ja Seo, and Ki-Min Bark ${ }^{\dagger}$ \\ Department of Chemistry, Chomam National Lniversitv, Guangit 500-757, Korea \\ "Department of Chemical Education and Institute of Katural Sciences, \\ Gyeongsang National Lniversity, Chini 660-701, Korea \\ Received JuIy 22, 2003
}

\begin{abstract}
The photochemical reactions of methanolic nalidixic acid (NAL) solution in the absence and in the presence of air have been investigated using $300 \mathrm{~nm}$ UV light. From the reactions. 1-ethyl-7-methyl-4-oxo-4-hydro-1.8naphthy ridine $(E M D N)$, formic acid and formaldehyde are produced. In the presence of air. hydrogen peroxide is also detected along with the products listed above. The presence of oxygen during the irradiation of methanolic NAL solution effects on the product yield. The initial quantum yields of the products and of the NAL decomposition are determined. Possible reaction pathways for the photochemical reaction are suggested on the basis of the products analysis.
\end{abstract}

Key Words : Photochemical reaction. Nalidixic acid. l-Ethỵl-7-methỵl-4-oxo-1.4-hỵdro-1.8-naphthỵridine. Formic acid. Formaldehyde

\section{Introduction}

Quinolone derivatives are the most frequently used antibacterial reagents. Nalidixic acid (NAL) is one of quinolone derivatives and was synthesized by Lesher et all. in $1962 .{ }^{\prime}$ A number of quinolone derivatives have been synthesized since then. However. it is reported that these drugs are able to induce photosensitive reactions in human skin by sunlight. $\hat{.}, 3$ It is, therefore, interesting to study the photochemical reaction of the quinolone antibactrial reagents since it could directly present the photoproducts during their UV-irradiation. However. their photochemical properties were poorly investigated. and only a few papers were reported on the study of the photochemical reaction of NAL

Dezer et $a l^{4}$ reported that photolysis of oxygen-free nalidixic acid in the basic solution results in the loss of the $\mathrm{COOH}$ group at the 3-position. From the decarboxylation. 1ethyl-7-methyl-4-oxo-1.4-hydro-1,8-naphthyridine (EMDN) is yielded. Carbon dioxide, ethylamine and 1-ethyl-1,4-dihydro7-methyl-2,4-dioxo-1.8-naphthyridine are also produced using a polychromatic UV light. Douglas et al. tested the photosensitization with nalidixic acid and oxolinic acids as photosensitizers in aqueous solution using $365 \mathrm{~mm}$ light. Verneersch et al. reported on the study of a laser flash photolysis. ${ }^{6}$ The $335 \mathrm{~nm}$ laser flash photolysis of nalidixic acid at $\mathrm{pH} 9.2$ leads to the triplet state formation of the nalidixate anion. In 1990. Fernandez et al. proposed the mechanism of photohaemolysis of EMDN. ${ }^{7}$ It is, however, hardly reported not only on the quantitative investigation on the photochemical reaction pathway of NAL but also on the photolysis of NAL in nonaqueous solution. NAL can be dissociated in aqueous solution and we reported its $\mathrm{pK}_{\mathrm{a}}$ value was found to be $6.33{ }^{8}$ It is conventional to postulate photochenical reaction pathway based on the product analysis because photochemical

Conesponding author. Tel: +82-62-530-3376; Fax: +82-62-5303389; e-mail: hparkiachomnam.ac.kr reaction undergoes very fast and various products can be formed. The purpose of this study is to elucidate the photochemical reaction pathway of methanolic NAL in the absence and in the presence of air using the UV light of $300 \mathrm{~nm}$ based on the products analy sis.

\section{Experimental Section}

Reagents and General. Nalidixic acid (NAL) was purchased from Sigma Chemical Co. (St. Louis, U.S.A.) and was used without further purification. The other chemicals were reagent grade and used as received. Methanol was first saturated by bubbling for 60 minutes with high purity argon and then 1 $\mathrm{mM}$ deaerated methanolic NAL solution was prepared. 80 $\mathrm{mL}$ of the prepared solution $(\mathrm{ImM})$ was transferred into the irradiation vessel and irradiated at room temperature using a photochemical reactor (Rayonet. RPR-208) equipped with a UV lamp as a monochromatic light source $(\lambda=300 \mathrm{rm})$.

The intensity of the lamp irradiance was measured by potassium ferrioxalate actinometry. ${ }^{9.11}$ The reduction yield of $\mathrm{Fe}^{+3}{ }_{\text {aq }}$ to $\mathrm{Fe}^{+\hat{3}}{ }_{\text {aq }}$ ion after irradiation of $6 \mathrm{mM}$ deaerated aqueous potassium ferrioxalate solution was measured by spectrophotometric method. ${ }^{11}$ The lanp intensity was found to be $7.66 \times 10^{18} \mathrm{~h} v \cdot \mathrm{L}^{-1} \mathrm{~min}^{-1}$ from the known quantum yield. $\mathrm{Q}\left(\mathrm{Fe}^{-{ }^{-2}}{ }_{\text {aq }}\right)=1.24 .^{9.15}$ No change in the intensity of the lamp irradiance was observed over the period of the experiment.

Steady-state fluorescence spectra of the $0.05 \mathrm{mM}$ deaerated NAL in methanol-water mixtures were obtained with a spectrofluorometer (Perkin-Elmer. LS- 50 ) at $25^{\circ} \mathrm{C}$. Methanolwater mixtures were prepared using doubly distilled water. which was obtained by passing distilled water through a deionization system (Barnstead, Nonopure II). Fluorescence quantum yields were determined using quinine sulfate as a reference. ${ }^{13-1+}$ The absorption spectra were taken using a UV-Vis spectrophotometer (Uvikon, model 943, Italy). 
Products Analysis. To perform the qualitative analysis of the products after irradiation of NAL in methanol, $60 \mathrm{~mL}$ of the irradiated solution was concentrated to $5 \mathrm{~mL}$ using a rotary vacuum evaporator. The sample prepared was then analyzed by two kinds of analytical methods. One of them was using a GC-MS system (Varian saturn; DB-5 capillary column $60 \mathrm{~m} \times 0.32 \mathrm{~m}$. El method); [product; $\mathrm{m} / \mathrm{z}(\mathrm{rel}$. intensity), formaldehyde; 28(10), 29(100), 30(35), formic acid; $17(20), 29(100), 44(40), 46(60)]$. Identifications and determinations were made by comparison with fragmentation patterns of known amounts of the pure substances. The other was analyzed using a LC-MS system (Mariner MSspectrometer equipped with ESI interface, capillary $\mathrm{C}_{18}$ reversed column, eluent: $1: 4$ volume ratio of acetonitrile : water). Two MS spectra were obtained: one has 232 molecular weight indicating NAL and the other has 188 molecular weight. These were separated by colunn chromatography (eluent was $I: 1$ volume ratio of benzene:ethylacetate). The solution was then washed by methanol and dried. The ${ }^{1} \mathrm{H}-$ NMR spectrum of the sample was obtained by a ${ }^{1} \mathrm{H}-\mathrm{NMR}$ spectrometer (Varian Gemini; $\mathrm{CDCl}_{3} .300 \mathrm{MH}$ ) $\delta 1.44(3 \mathrm{H}$, $\mathrm{t}, J=7.2 \mathrm{~Hz}), 2.44(3 \mathrm{H}, \mathrm{s}), 4.40(2 \mathrm{H}, \mathrm{q}, J=7.2 \mathrm{~Hz}), 6.30$ $(1 \mathrm{H}, \mathrm{d}, J=7.8 \mathrm{~Hz}), 7.19(1 \mathrm{H}, \mathrm{d}, J=8.1 \mathrm{~Hz}), 7.66(1 \mathrm{H}, \mathrm{d}$, $J=7.8 \mathrm{~Hz}) .8 .58(1 \mathrm{H}, \mathrm{d}, J=8.1 \mathrm{~Hz})$ and it was characterized to be 1-ethyl-7-Inethyl-4-oxo-1.4-hydro-1,8-naphthyridine (EMDN).

Quantitative analysis of the products was carried out either by the liquid chromatographic method or by the spectrophotometric method because the gas chromatographic method has a poor reproducibility. Quantitative analysis of EMDN produced and NAL decomposed performed by a liquid chromatography (Young-Lin. Model 930; phase sep S5 ODS2 column, eluent: 30 vol. $\%$ of $2 \mathrm{mM}$ aqueous $\mathrm{KH}_{2} \mathrm{PO}_{4}$ solution, $\mathrm{UV}$ detecter) with a calibration curve using pyridine as an internal standard. On the other hand, the amount of formic acid was determined by the same liquid chromato-graphy equipped with a Ion-Exclusion column (IC-PAK ${ }^{\text {TN. }}$; eluent: $0.01 \mathrm{~N} \mathrm{H}_{2} \mathrm{SO}_{4}$, UV detecter) using citric acid as an internal standard. The amount of formaldehyde produced was determined by spectrophotometric method. ${ }^{15}$ An aliquot $(5 \mathrm{~mL})$ of the irradiated solutions treated with Hantz reagent. The molar extinction coefficient $(\varepsilon)$ of colored complex was $7860 \mathrm{M}^{-1} \mathrm{~cm}^{-1}$ at $412 \mathrm{~nm}$ in this experiment and it was not interfered by the presence of the other compounds such as methanol. Spectrophotometric determination of hydrogen peroxide was performed by treatment with acidic $\mathrm{CiCl}_{4}$ solution. ${ }^{16}$ The developed color was characterized by the absorbance maximum at $414 \mathrm{~nm}$ and its molar extinction coefficient $(\varepsilon)$ at this wavelength was determined to be $3430 \mathrm{M}^{-1} \mathrm{~cm}^{-1}$.

\section{Results and Discussion}

Irradiation of $1.0 \mathrm{mM} \mathrm{NAL}$ in methanol was carried out using $300 \mathrm{~nm} U \mathrm{~V}$ light in the absence and in the presence of air. 'lo examine the photochemical reaction. UV-Vis absorption spectra and $\mathrm{pH}$ values were measured before and after

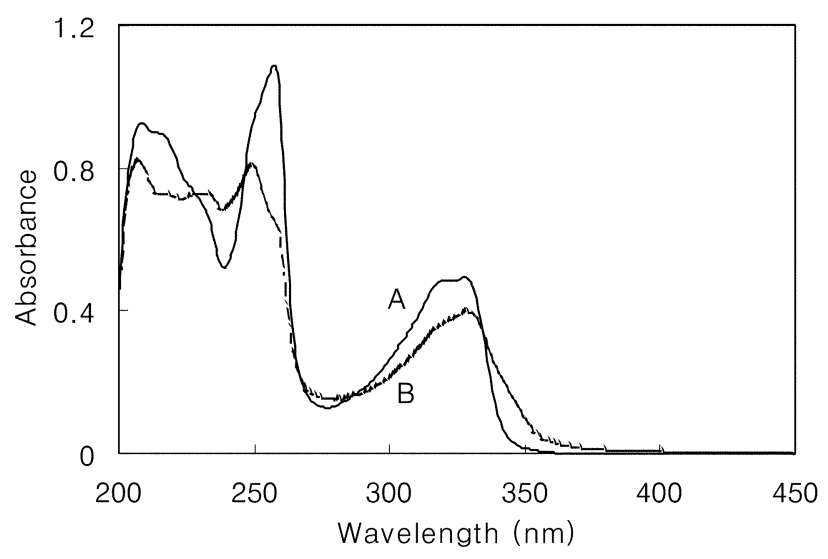

Figure 1. UV-Vis absomption spectra betore (A) and after (B) irradiation of I m. N NAL in methanol: applied UV dose for irradiation $(\lambda=300 \mathrm{~nm}): 6.89 \times 10^{30} \mathrm{~h} v \cdot \mathrm{mL}^{-1}$.

irradiation of methanolic NAL solution. As shown in Figure I, their absorption spectra appeared as a similar shape in the wavelength region above $260 \mathrm{~nm}$ whereas they were different in the wavelength region below $260 \mathrm{~nm}$. The absorption spectrum of aqueous NAL solution contains two bands in the wavelength region above $260 \mathrm{~nm}$, indicating two chromophores: one assigned from the nitrogen atom at the position 1 to carboxyl group and the other from the methyl group attached to the 7-carbon atom to carbonyl group. ${ }^{8}$ The fact that the absorption spectra appeared as a similar shape in the wavelength region above $260 \mathrm{~nm}$ may imply that aromatic moiety in NAL was not so greatly altered after irradiation of $\mathrm{NAL}$ using $300 \mathrm{~nm}$ UV light. The $\mathrm{pH}$ value of the solution decreased from 6.59 to 5.43 when $6.89 \times 10^{20} \mathrm{~h} v^{\prime} \mathrm{mL}^{-1} \mathrm{UV}$ dose were irradiated in the deaerated methanolic NAL solution. The same $\mathrm{pH}$ change occurred in the irradiation of aerated methanolic NAL solution, but its difference $(0.71)$ was rather small compared to that of deaerated solution. It indicates that some acids were produced in the irradiation of methanolic NAL solution and oxygen affects the photochemical reaction process. After irradiation of the solution, 1-ethyl-7methyl-4-oxo-4-hydro-1,8-naphthyridine (EMDN), formic acid and formaldehyde were mainly produced. The $\mathrm{pH}$ change of the solution before and after irradiation could be explained by the formation of formic acid.

The photolysis of $1.0 \mathrm{mM}$ deaerated as well as aerated methanolic NAL solution was studied as a function of the number of quanta. As shown in rigure 2, the decomposition of NAL in methanol decreased but not in proportion to the number of quanta. The electronically excited NAL is not so much decomposed and it has some photostability in the initial state of the photochemical reaction. However, the decomposition of NAL increased regardless of the absence and the presence of air, as the number of quanta increased. This behavior indicates that some secondary photochemical reactions, which contribute to the decomposition of NAL. occurred in the system by the absorption of the UV light. In addition to this, the amounts of photoproducts such as EMDN, formic acid, and formaldehyde did not increased in proportion to the number of quanta as presented in Figure 3 . 


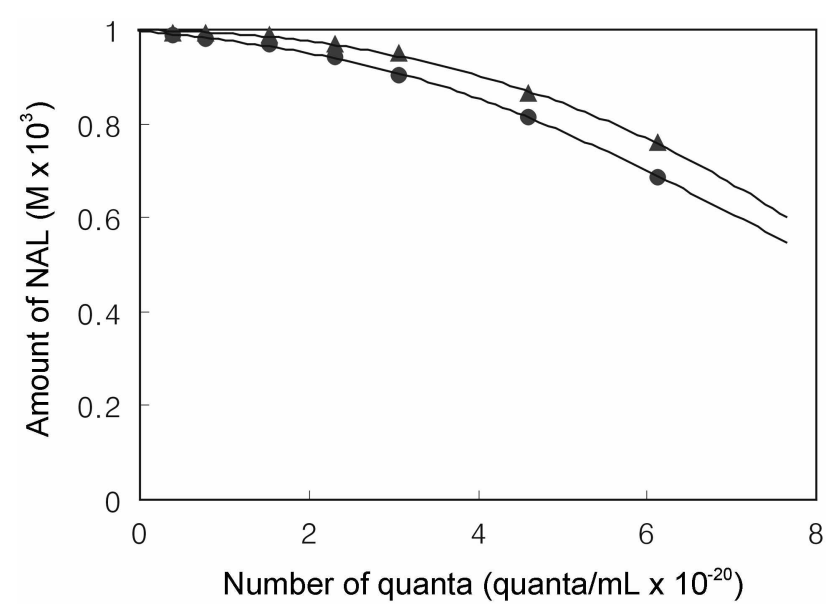

Figure 2. Amount of $\mathrm{XAl}$. remained after irradiation of $1.0 \mathrm{mM}$ methanolic NAI as a lunction of the number of cquanta: $(\bullet)$ in the absence of air: $(\Delta)$ in the presence of air.

Therefore, to avoid the interference of secondary reactions, we determined the initial quantum yields $\left(Q_{i}\right)$, obtained from the slope of tangent line of the curve shown in Figure 3. The results are sumınarized in Table $\mathrm{l}$.

In the irradiation of NAL in methanol by $300 \mathrm{~nm}$ UV light, methanol does not absorb the light and only NAL absorbs all

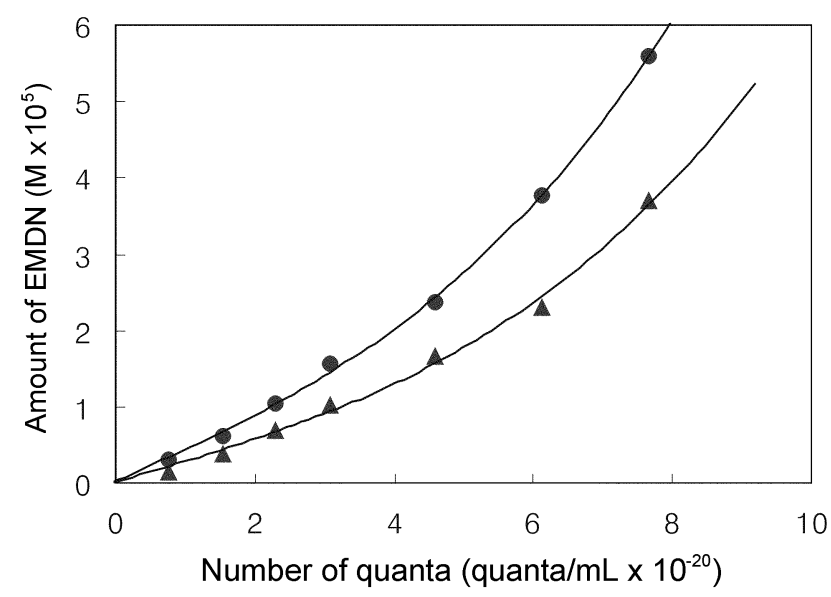

Figure 3. Iormation of EMDX after joradiation of $1.0 \mathrm{mM}$ methanolic NAL as a function of the number of quanta: $(\bullet)$ in the absence of air: $(\Delta)$ in the presence of air.

Table I. Initial quantum yield $\left(Q_{i}\right)$ of the compounds after iradiation ( $\lambda \quad 300 \mathrm{~nm}$ ) of $1 \mathrm{mM}$ nalidixic acid (.ML) in aeraled and deacrated methanol

\begin{tabular}{lcc}
\hline \multirow{2}{*}{ Compounds } & \multicolumn{2}{c}{ Q when saturated with } \\
\cline { 2 - 3 } & $\Lambda \mathrm{r}$ & Air \\
\hline NAL & $5.51 \times 10^{5}$ & $2.36 \times 10^{5}$ \\
FMIN & $2.47 \times 10^{5}$ & $1.46 \times 10^{5}$ \\
Formic acid & $2.53 \times 10^{5}$ & $4.19 \times 10^{\circ}$ \\
Formaldehyde & $2.31 \times 10^{5}$ & $2.22 \times 10^{5}$ \\
Ilydrogen peroxide & - & $\leq 10^{\circ}$ \\
\hline
\end{tabular}

"These values indicate the initial quantum yield of nalidixic acid deconposed. The others indicate the initial quantum yield of the products.

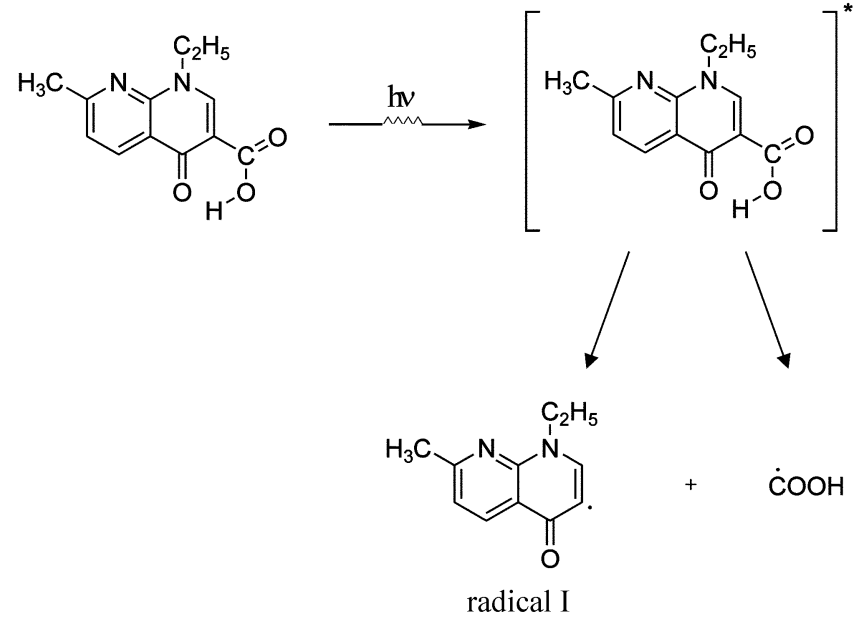

Scheme I

of the light at the initial process of the reaction. It means that the photochemical decomposition of NAL in methanol begins with absorption of the light by NAL. The fact that EMDN and formic acid are mainly produced in the irradiation of the solution, is an evidence that the electronically excited NAL by the absorption of $300 \mathrm{~nm}$ is predominantly decomposed by splitting into the radical $\mathrm{I}$ and $\mathrm{COOH}$ radical as in Scheme 1 .

The radicals of I and $\mathrm{COOH}$ produced can react with the chemical species in the solution. However, most of the radicals attack methanol because methanol has the largest concentration among the chemical species in the solution, and its reaction probability of the reactions defined by multiplication of concentration and rate constant is greatest. Hence, the formation of EMDN and formic acid could be explained by the reactions ( 1 ) and (2), respectively.

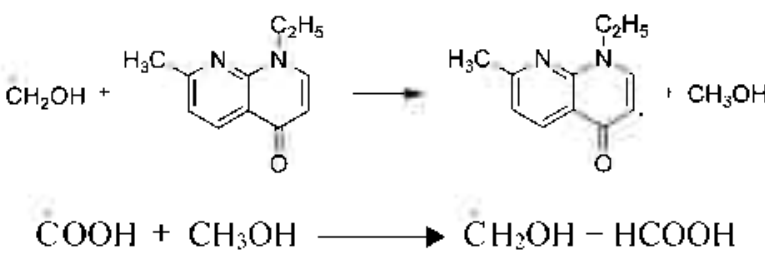

The initial quantum yield of EMDN and that of formic acid produced by the irradiation of deaerated methanolic NAL were found to be nearly same. It supports that the hypothesis described above is reasonable because the products were formed by the radicals of $I$ and $\mathrm{COOH}$, and the amount of each radical produced by the photofragmentation of NAL was same. The photochemical reaction mechanism for the formation of EMDN is quite similar to that proposed by Dezer $e t a l^{4}$ although they reported on the photolysis of oxygen-free nalidixic acid in the basic solution leading to the formation of the dissociated chemical species of NAL. so called nalidixate anion. However, NAL is hardly dissociated in methanol. It means that the photolysis of NAL in methanol was proceeds from its molecular state rather than from its anionic form. This is the reason why formic acid 
Table 2. Stokes' shift $(\Delta v)$ and fluorescence quantum yields $\left(Q_{F}\right)$ of NAL in the irradiation of $0.05 \mathrm{mM}$ nalidisic acid (NAL) in deaerated methanol-water mixtures: $\lambda_{\mathrm{x}}=300 \mathrm{~mm}$

\begin{tabular}{ccccc}
\hline Vol. \% of $\mathrm{H}_{2} \mathrm{O}$ & $\mathrm{v}_{\mathrm{a}}\left(\mathrm{cm}^{-1}\right)^{a}$ & $v_{\mathrm{f}}\left(\mathrm{cm}^{-1}\right)^{a}$ & $\Delta v\left(\mathrm{~cm}^{-1}\right)^{b}$ & $Q_{F}{ }^{c}$ \\
\hline 100 & 30289 & 26826 & 3463 & 0.0054 \\
95 & 30717 & 27049 & 3668 & 0.0095 \\
90 & 30741 & 27135 & 3606 & 0.0108 \\
75 & 30822 & 27218 & 3604 & 0.0115 \\
50 & 30746 & 27350 & 3396 & 0.0114 \\
25 & 30699 & 27405 & 3294 & 0.0110 \\
10 & 30625 & 27395 & 3230 & 0.0113 \\
5 & 30621 & 27394 & 3227 & 0.0103 \\
0 & 30569 & 27399 & 3170 & 0.0100 \\
\hline
\end{tabular}

"Energy of absorption $\left(\nu_{a}\right)$ or fluorescence center of graxity $\left(v_{0}\right)$ : uncertainty is $\pm 60 \mathrm{~cm}^{-1} . \Delta v^{b}=v_{\mathrm{d}}-\mathrm{v}_{\mathrm{f}}$. 'Fluorescence quantum vields: uncertainty is $\leq 6^{\circ} \mathrm{i}$.

was formed by the irradiation of NAL in methanol.

In general. the electronically excited state of aromatic compounds possesses larger dipole moment $(\mu)$ than that of the ground state $(\mu)$. Therefore. it might be expected that the electronically excited NAL in methanol could be dissociated to nalidixate anion form more easily. To ascertain this expectation, we measured the spectroscopic properties such as Stokes shift and fluorescence quantum yield of NAL in the methanol-water mixtures. As shown in Table 2, Stokes shift of NAL decreased as the polarity of the mixtures decreased. This tendency is very general because the emission spectrum shifts to shorter wavelengths as the solvent polarity decreases. However. the fluorescence quantum yields of NAL were independent in the methanol-water mixtures except for that in water $\left(\mathrm{Q}_{\mathrm{F}}=0.0054\right)$. It indicates that the chemical structure of the electronically excited NAL in water was different from that in mixtures with methanol. If NAL could be dissociated to nalidixate anion form, the amount of fluorophore should be changed by the polarity of the mixtures and fluorescence quantum yield of NAL should be also changed. But. it was found to be nearly same value. This result could be interpreted by the formation of intramolecular hydrogen bond between the proton of the carboxyl group and the keto oxygen in NAL. This is a reason why carboxyl group in NAL was not dissociated in the electronically excited state and why $\mathrm{COOH}$ radical could be produced by' the irradiation of NAL in methanol.

The $\mathrm{C}_{3} \mathrm{H}_{3} \mathrm{OH}$ radical formed by the processes (1) and (2) can predominantly attack methanol. Sonntag reported that -hy'drogen atom in methanol is mainly dissociated by attack of a radical. ${ }^{17}$ It is therefore expected that $\dot{\mathrm{C}}^{\mathrm{C}} \mathrm{H}_{2} \mathrm{OH}$ radical is reproduced by the reaction of a $\mathrm{CH}_{2} \mathrm{OH}$ radical and methanol. Hence the $\mathrm{CH}_{2} \mathrm{OH}$ radicals should be stabilized by the another reaction pathway such as the combination with each other to produce formaldehyde and ethylene glycol as in reaction (3). Therefore the formation of formaldehyde could be interpreted by the reaction (3a)

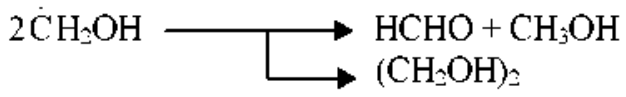

The initial quantum yields of formaldehyde and that of formic acid produced by the irradiation of NAL were found to be nearly same. It supports that the hypothesis described above is reasonable because formaldehyde is formed by disproportionation process of $\dot{\mathrm{C}} \mathrm{H}_{2} \mathrm{OH}$ radical which is produced twice as the amount of $\dot{\mathrm{COOH}}$ radical produced in the irradiation of NAL.

In the presence of air. As presented in Table 1. the initial quantum yield of the NAL decomposed in the presence of air is smaller than that in the absence of air. In addition to this, the initial quantum yields of the EMDN and formic acid produced in the presence of air are smaller than those in the absence of air. It means that oxygen affects the photolysis of NAL in methanol. Decomposition of NAL was carried out by direct photolysis with $300 \mathrm{~nm}$ UV light. It is well known that oxygen is a good dynamic quencher and the electronically excited NAL can transfer its energy to oxygen very fast in the presence of air. As a result the population of electronically excited NAL might be diminished and the amount of NAL decomposed also decreases. Since the formation of EMDN and formic acid is caused by radicals of I and $\dot{\mathrm{C}} \mathrm{OOH}$. and the same amount of the radicals are produced by the photolysis of NAL, their initial quantum yields should be nearly same. However, the initial quantum yield of formic acid is smaller than that of EMDN. It indicates that there is another reaction pathway. Wine et $a l^{18}$ proposed that $\mathrm{COOH}$ radical could be combined with oxygen and it is converted into $\mathrm{CO}_{2}$ and $\mathrm{HO}_{2}$ radical as in reaction (4).

$$
\dot{\mathrm{COOH}}+\mathrm{O}_{2} \longrightarrow \mathrm{CO}_{2}+\mathrm{H}_{2}
$$

The $\mathrm{HO}_{2}$ radical formed in reaction (4) is so unstable that it can attack methanol to produce hydrogen peroxide and $\mathrm{CH}_{2} \mathrm{OH}$ as in reaction (5).

$$
\mathrm{H}_{2} \mathrm{O}+\mathrm{CH}_{3} \mathrm{OH} \longrightarrow \mathrm{H}_{2} \mathrm{O}_{2}+\dot{\mathrm{C}}_{2} \mathrm{H}_{2} \mathrm{OH}
$$

The $\dot{\mathrm{C}}_{2} \mathrm{OH}$ radical produced in reaction (5) takes part in the formation of formaldehyde again. Therefore the initial quantum yield of formaldelyde is nearly same value regardless of existence of air. To test this expectation. the amount of lydrogen peroxide formed by the irradiation of aerated NAL in methanol was analyzed. The formation of hy'drogen peroxide produced by the irradiation of aerated NAL in methanol was detected. But its amount was so small. It is because the formation of $\mathrm{HO}_{2}$ radical is also small. In contrast to the results. its formation was not detected in the irradiation of deaerated methanolic NAL.

\section{Conclusions}

1-Ethyl-7-methyl-4-oxo-4-lydro-1.8-naphthyridine (EMDN). formic acid. and formaldelyde were produced as a main product in the irradiation of NAL in methanol. The electronically excited NAL in methanol by the absorption of 300 nm UV light exists its molecular form rather than its dissociated form, and it is mainly splitted into its decarbox- 
ylated radical and $\mathrm{COOH}$ radical. The both radicals attack methanol to form EMDN and formic acid. Thus $\mathrm{C}_{2} \mathrm{H}_{2} \mathrm{OH}$ radical is produced and the formation of formaldelyde could be explained by the disproportionation process of the radical. The photochenical reaction of NAL was affected by the presence of oxygen in the solution. The decomposition of NAL as well as the formation of EMDN and formic acid better proceeded in the absence of air than in the presence of air. However. the amount of formaldehyde produced is not affected by the presence of air because $\mathrm{HO}_{2}$ radical formed by the reaction between $\dot{\mathrm{C}} \mathrm{OOH}$ radical and oxygen contributed to the formation of $\dot{\mathrm{C}}_{2} \mathrm{H}_{2} \mathrm{OH}$ radical.

\section{References}

1. Lesher G.; Froelich. M.: Grlett. I. J. Med. Pharm. Chem. 1962. 5 , 1063.

2. Kang. J. S.: Kim. T. H.: Park. K. B.: Chung. B. H.: Youn. J. I. Photodermal Photoinmtinol Photone. 1993. 9.159.

3. Cho, Y. H.: Kim. T. H: Park, H. B: Park C. K : Park. K. M. Kor J. Dermatol. 1995, 33. 1021.
4. Detzer. N.: Huber, B. Tetrahedron 1975. 31, 1937.

5. Moore. D. E.: Hemmens. V. J.: Yip. H. Photochent Photobiol. 1984.39 .57$.

6. Vermeersch. G.: Ronfard-Haret. J. C.: Bazin. M.: Carillet. V: Moriere, P.: Santus, R. Photochem and Photobiol 1991, 54. 661 .

7. Fernandez, E; Cardenas. A. M. d. Photochem Photobiol. B: Photobiol. 1990. +. 329.

8. Park. H. R.: Chun1g. K. Y.: Lee. H. C.: Lee. J. K.: Bark. K. M. Bull. Korean Chent. Soc. 2000. 21. 849.

9. Hatchard, C. G.; Parker. C. A. Proc. Roy Soc. (Londonj 1956. A235. 518 .

10. Lee. J.: Seliger, H. H. J. Chem Phys. 1968. 40.519.

11. Atkins. R. C.J. Chem. Ed 1975.52.550.

12. Bark. K. M.: Force. R. K. Spectrochim. Acta 1993. 19.4 . 1605.

13. Eaton. D. F. Reference Contpounds for Fluorescence Mecastrenents: IUPAC Organic Chemistry Division: Washington DC, USA. 1987: pp 1-11.

14. Demas. J. N.: Grosby, G. A. J. Phus. Chem. 1971, 75. 2463

15. Nasch. T. Biochem. J. 1958. 55.418.

16. Wolfe. W. C. J. Anal. Chem. 1962. 3t. 1328.

17. Sonintag. C. Z. Phus Chent 1970. 69. 292.

18. Wine. P. H.: Astalos. R. J.: Mauldin III, R. L. J. Plys. Chem 1985. 89.2620 . 